\title{
A CANONICAL LINEAR ORDER FOR THE MAXIMAL CHAINS OF A TREE
}

\author{
JAMES C. OWINGS, JR. ${ }^{1}$
}

A tree $T$ is a pair $\left(\leqq_{T}, \hat{T}\right)$ where $\leqq_{T}$ is a partial ordering on $\hat{T}$ (the field of $T$ ) with the property that if $x, y, z \in \hat{T}, x \leqq{ }_{T} z$, and $y \leqq_{T} z$, then either $x \leqq_{T} y$ or $y \leqq_{T} x$. Notice that $T$ may have more than one "component." Points $x, y$ of $T$ are comparable if $x \leqq_{T} y$ or $y \leqq_{T} x$; otherwise, $x$ and $y$ are incomparable. A chain is a subset of $\hat{T}$ any two points of which are comparable; a cochain is a subset of $\hat{T}$ any two points of which are incomparable. A maximal chain is a subset $C$ of $\hat{T}$ such that $C$ is a chain of $T$ but is not a proper subset of any chain of $T$.

Suppose now that $T$ is a tree whose field $\hat{T}$ is well ordered; in fact, suppose $\hat{T}$ has cardinality $\kappa$. We show how this $\kappa$-ordering induces in a natural way a linear ordering on the maximal chains of $T$ which preserves much of the original tree structure. Thus it is superior to the ordinary lexicographic ordering, which would commonly preserve none of the structure. We then use our ordering to give a new proof of Miller's Theorem, a remarkable result related to the Suslin problem.

We have assumed $\hat{T}$ is well ordered by the ordinals less than $\kappa$; if $\alpha<\kappa$ let $x(\alpha)$ be the " $\alpha$ th" point of $\hat{T}$. If $A$ is a nonempty subset of $\hat{T}$, let $q(A)$ be the least ordinal $\alpha<\kappa$ for which there exists a $y \in \hat{T}$ such that $y \leqq_{T} x(\alpha)$ and $y \in A$. If $C, C^{\prime}$ are distinct maximal chains, we define $C<{ }_{L} C^{\prime}$ if and only if $q\left(C-C^{\prime}\right)<q\left(C^{\prime}-C\right)$. One easily shows, using the fact $T$ is a tree, that, for distinct maximal chains $C$ and $C^{\prime}$, $q\left(C-C^{\prime}\right) \neq q\left(C^{\prime}-C\right)$. Thus any two distinct maximal chains are comparable w.r.t. the ordering $<_{L}$.

That $<_{L}$ is transitive is not at all obvious. Let $C_{1}, C_{2}, C_{3}$ be three maximal chains and suppose $C_{1}<_{L} C_{2}, C_{2}<{ }_{L} C_{3}$. We must show $C_{1}<{ }_{L} C_{3}$. Suppose, to the contrary, that $C_{3}<{ }_{L} C_{1}$; i.e., assume $q\left(C_{3}-C_{1}\right)$ $<q\left(C_{1}-C_{8}\right)$. Using standard Venn diagram techniques, one derives the set-theoretic identities

$$
\begin{aligned}
& C_{1}-C_{3}=\left(\left(C_{1}-C_{2}\right)-\left(C_{3}-C_{2}\right)\right) \cup\left(\left(C_{2}-C_{3}\right)-\left(C_{2}-C_{1}\right)\right), \\
& C_{3}-C_{1}=\left(\left(C_{3}-C_{2}\right)-\left(C_{1}-C_{2}\right)\right) \cup\left(\left(C_{2}-C_{1}\right)-\left(C_{2}-C_{3}\right)\right) .
\end{aligned}
$$

CASE 1. $q\left(C_{3}-C_{1}\right)=q\left(\left(C_{3}-C_{2}\right)-\left(C_{1}-C_{2}\right)\right)$. Then, since $q\left(C_{3}-C_{2}\right)$ $\leqq q\left(\left(C_{3}-C_{2}\right)-\left(C_{1}-C_{2}\right)\right)$ and $C_{2}<_{L} C_{3}, \quad q\left(C_{2}-C_{3}\right)<q\left(C_{3}-C_{1}\right)$. If

Received by the editors August 7, 1967.

1 The research for this paper was done while the author held a National Science Foundation Graduate Fellowship at Cornell University. 
$q\left(C_{2}-C_{3}\right)<q\left(C_{2}-C_{1}\right)$, we would have $q\left(\left(C_{2}-C_{3}\right)-\left(C_{2}-C_{1}\right)\right)=$ $q\left(C_{2}-C_{3}\right)$, yielding $q\left(C_{1}-C_{3}\right)<q\left(C_{3}-C_{1}\right)$, contrary to assumption. So $q\left(C_{2}-C_{1}\right) \leqq q\left(C_{2}-C_{3}\right)$. Since $C_{1}<{ }_{L} C_{2}$, this gives $q\left(C_{1}-C_{2}\right)<q\left(C_{2}-C_{3}\right)$. If $q\left(C_{1}-C_{2}\right)<q\left(C_{3}-C_{2}\right)$, we would have $q\left(\left(C_{1}-C_{2}\right)-\left(C_{3}-C_{2}\right)\right)$ $=q\left(C_{1}-C_{2}\right)$, yielding $q\left(C_{1}-C_{3}\right) \leqq q\left(C_{1}-C_{2}\right)<q\left(C_{2}-C_{3}\right)<q\left(C_{3}-C_{1}\right)$, again contrary to assumption. So $q\left(C_{3}-C_{2}\right) \leqq q\left(C_{1}-C_{2}\right)<q\left(C_{2}-C_{3}\right)$, contradicting the hypothesis $C_{2}<{ }_{L} C_{3}$.

CASE 2. $q\left(C_{3}-C_{1}\right)=q\left(\left(C_{2}-C_{1}\right)-\left(C_{2}-C_{3}\right)\right)$. The argument is similar to that for Case 1. Interchange $C_{1}-C_{2}$ with $C_{2}-C_{3}, C_{2}-C_{1}$ with $C_{3}-C_{2}$, and $C_{1}<{ }_{L} C_{2}$ with $C_{2}<{ }_{L} C_{3}$.

Since one of the above two cases must hold, we have reached a contradiction. So $<_{L}$ is a linear order for the maximal chains of $T$. One sees that if $C, C^{\prime}, C^{\prime \prime}$ are maximal chains such that $C \cap C^{\prime} \cap C^{\prime \prime}$ is a proper subset of $C^{\prime} \cap C^{\prime \prime}$, then $C<{ }_{L} C^{\prime}$ iff $C<{ }_{L} C^{\prime \prime}$.

We offer the following application. A Suslin continuum is a continuous (complete and dense) nonempty linear order without endpoints with the property that every disjoint collection of open intervals is countable. So, in particular, the real numbers form a Suslin continuum. A $\kappa$-Suslin tree (where $\kappa$ is a cardinal) is a tree whose field has cardinality $\kappa$ and each of whose chains and cochains has cardinality less than $\kappa$. In 1920 [2] Suslin posed the famous problem "Is every Suslin continuum isomorphic to the ordering of the reals?." In 1943 [1] Miller showed that there exists a Suslin continuum not isomorphic to the reals if and only if there exists an $\aleph_{1}$-Suslin tree. The "only if" direction is easy but uses the axiom of choice. The "if" direction is much more difficult but avoids the axiom of choice by capitalizing upon the well-ordering hypothesized for the field of the tree.

S. Tennenbaum [3] and T. Jech [4] used the forcing method of P. J. Cohen to construct a model of Zermelo-Fraenkel set theory (ZF) and the Axiom of Choice (AC) in which there exists an $\aleph_{1}$-Suslin tree. More recently he and $\mathrm{R}$. Solovay have completed the proof of independence; that is they have found a model of $\mathrm{ZF}$ and $\mathrm{AC}$ in which there is no $\boldsymbol{N}_{1}$-Suslin tree. Thus, most aspects of the Suslin problem have been settled, with Miller's Theorem as a central lemma.

We now use our method to give a simple construction for the difficult half of Miller's Theorem. Let $S$ be an $\boldsymbol{N}_{1}$-Suslin tree. Let $C$ be the set of points $c$ of $\hat{S}$ such that $\left\{x \mid x \in \hat{S}\right.$ and $\left.c \leqq s_{s} x\right\}$ is countable. One can show that $C$ is countable. For if $c, c^{\prime} \in C$ then $c, c^{\prime}$ belong to the same "component" of $C$ iff there exists a point $c^{\prime \prime} \in C$ such that $c^{\prime \prime} \leqq{ }_{S} C$ and $c^{\prime \prime} \leqq{ }_{S} c^{\prime}$. Since every cochain of $S$ is countable, $C$ has only countably many components. And since every chain of $S$ is countable, 
each component of $C$ is countable. Let $S^{\prime}$ be the subtree of $S$ with field $\hat{S}-C$. Then $S^{\prime}$ is also an $\boldsymbol{N}_{1}$-Suslin tree and every point of $S^{\prime}$ belongs to uncountably many maximal chains of $S^{\prime}$.

Let $L$ be our linear ordering of the maximal chains of $S^{\prime}$. Now it is easy to see that, if $C, C^{\prime}$ are maximal chains of $S^{\prime}$ then $\left\{x \mid C<{ }_{L} x<{ }_{L} C^{\prime}\right\}$ is either uncountable or is empty. This is because every member of $S^{\prime}$ belongs to uncountably many maximal chains, so that if there is one such $x$ there must be uncountably many. Call $C \sim C^{\prime}$ if $\left\{x \mid C<_{L} x<_{L} C^{\prime}\right.$ or $\left.C^{\prime}<_{L} x<_{L} C\right\}=\varnothing . \sim$ is then an equivalence relation, with each equivalence class containing at most two maximal chains. Furthermore, it is obvious that $<_{L}$ is well defined w.r.t. $\sim$. Thus we obtain a new linear ordering $L^{\prime}$, on the equivalence classes. The necessity for "closing the gaps" in $L$ is analogous to the necessity for equating the Cauchy sequences $.5000 \cdots$ and $.4999 \cdots$.

$\mathrm{L}^{\prime}$ is obviously dense. If $C$ is a maximal chain of $S^{\prime}$, let $[C]=\left\{C^{\prime} \mid C^{\prime} \sim C\right\}$. The two arguments which follow use only the basic property of $<_{L}$; namely, if $C \cap C^{\prime} \cap C^{\prime \prime}$ is a proper subset of $C^{\prime} \cap C^{\prime \prime}$, then $C<{ }_{L} C^{\prime}$ iff $C<{ }_{L} C^{\prime \prime}$ (and so also $C>{ }_{L} C^{\prime}$ iff $C>{ }_{L} C^{\prime \prime}$ ).

The fact every cochain of $S^{\prime}$ is countable may be used to show that any collection of disjoint intervals of $L^{\prime}$ is countable. For let $V$ be such a collection. Let $I \in V$. Then $I=\left([C],\left[C^{\prime}\right]\right)$, where $C<_{L} C^{\prime}$ and $\left\{x \mid C<{ }_{L} x<{ }_{L} C^{\prime}\right\}$ is uncountable. Let $x(\alpha)$ be the $\alpha$ th point of $S^{\prime}$ and let $\beta$ be the least $\alpha$ for which there exist chains $C_{1}, C^{*}, C_{2}$ satisfying $C<{ }_{L} C_{1}<{ }_{L} C^{*}<{ }_{L} C_{2}<{ }_{L} C^{\prime}, x(\alpha) \in C^{*}-\left(C_{1} \cup C_{2}\right)$, and set $p(I)=x(\beta)$. Then for all chains $C^{\prime \prime}, p(I) \in C^{\prime \prime}$ implies $C_{1}<{ }_{L} C^{\prime \prime}<{ }_{L} C_{2}$. Thus, for all chains $C^{\prime \prime}, p(I) \in C^{\prime \prime}$ implies $[C]<_{L^{\prime}}\left[C^{\prime \prime}\right]<_{L^{\prime}}\left[C^{\prime}\right]$. So if $I, I^{\prime} \in V$ and $I \neq I^{\prime}$, then, because $I \cap I^{\prime}=\varnothing, p(I)$ and $p\left(I^{\prime}\right)$ are incomparable. Hence $\{p(I) \mid I \in V\}$ is a cochain of $S^{\prime}$ and is therefore countable. So $V$ is also countable.

Also, the fact every chain of $S^{\prime}$ is countable implies there is no countable subset of $L^{\prime}$ which is dense in $L^{\prime}$ (a subset $A$ of $L^{\prime}$ is dense in $L^{\prime}$ iff $A$ contains some point of every open interval of $\left.L^{\prime}\right)$. For suppose $A$ is a countable subset of $L^{\prime}$ and set $W=U\{C \mid[C] \in A\}$. Since every chain of $S^{\prime}$ is countable, $W$ is countable and so $T=S^{\prime}-W$ is an $\aleph_{1}$-Suslin tree. Let $p \in \hat{T}$ and let $D, D^{*}, D^{\prime}$ be distinct maximal chains of $T$ such that $p \in D \cap D^{*} \cap D^{\prime}$. Let $P=\left\{c \mid c \in S^{\prime}\right.$ and $\left.c<s^{\prime} p\right\}$ and set $C=D \cup P, C^{*}=D^{*} \cup P, C^{\prime}=D^{\prime} \cup P . C, C^{*}, C^{\prime}$ are maximal chains of $S^{\prime}$ and thus are linearly ordered by $<_{L}$; we may assume $C<_{L} C^{*}<_{L} C^{\prime}$. Then $[C]<_{L^{\prime}}\left[C^{\prime}\right]$ and for all chains $C^{\prime \prime},[C]<_{L^{\prime}}\left[C^{\prime \prime}\right]<_{L^{\prime}}\left[C^{\prime}\right]$ implies $p \in C^{\prime \prime}$. So, since $p \notin W, A$ is not dense in $L^{\prime}$.

Let $L^{\prime \prime}$ be the completion of $L^{\prime}$; that is, let $L^{\prime \prime}$ be the set of Dedekind cuts of $L^{\prime}$ under the ordering induced by $L^{\prime}$. The three properties 
of $L^{\prime}$ mentioned in the three foregoing paragraphs are inherited by $L^{\prime \prime}$. Finally, delete the endpoints (if any) of $L^{\prime \prime}$ to obtain the suborder $R . R$ is then a Suslin continuum which, because it contains no countable subset dense in itself, is not isomorphic to the reals. One easily convinces himself that there is no use of the axiom of choice in the above argument.

\section{REFERENCES}

1. Edwin W. Miller, $A$ note on the Souslin problem, Amer. J. Math. 65 (1943), 673-678.

2. M. Suslin, Problem 3, Fund Math. 1 (1920), 223.

3. S. Tennenbaum, Suslin's problem, Proc. Nat. Acad. Sci. U.S.A. 59 (1968), 60-63.

4. T. Jech, Non-provability of Souslin's hypothesis, Comment. Math. Univ. Carolinae 8 (1967), 291-305.

UNIVERSITY OF MARYLAND 